\title{
On Evolution of Some Associated Type Ruled Surfaces
}

\author{
Alev Kelleci* and Kemal Eren
}

\begin{abstract}
In this study, we define three new type ruled surfaces obtained by using the evolution of involute-evolute curve pair in Euclidean 3-space. Then, we give some nice results for the Gaussian curvature and the mean curvature of these surfaces. Moreover, considering the given involute-evolute curve pair, the graphics of these new type surfaces were drawn with the help of Mathematica.

Keywords: Ruled surface; evolution of space curve; inextensible surface; involute-evolute curve pair; Moving surface.

AMS Subject Classification (2020): Primary: $53 A 05$; Secondary: 53A25; 58C27.

${ }^{*}$ Corresponding author
\end{abstract}

\section{Introduction}

The theory of surface in three dimensional Euclidean space is widely used in different branches of science, particularly mathematics (differential geometry, topology, Partial Differential Equations (PDEs)), theoretical physics (string theory, general theory of relativity), graphic design and different branches of engineering. One of the most important of these surfaces is the ruled surfaces obtained by the movement of a line along the curve [1]. Also, various researches have been made considering the characterization of the ruled surfaces, their geometrical and algebraic properties $[2,3,7,8]$. Curves theory is also an important research subject of differential geometry like surfaces. Many studies have been done on the evolution of curves, the geometric characterization of curves and the evolution of inelastic plane curves [9-13, 29]. Many researchers have studied ruled surfaces and surfaces from these curves. For example, in the Euclidean space, the evolution of special ruled surfaces, evolution of translation surfaces, special ruled surfaces using quasi frame and ruled surfaces using a modified orthogonal frame were examined [14-21, 28]. One of the important curve pairs of curve theory is involute-evolute curves. In 1658, the concept of involute was first mentioned by Christian Huyhens, who conducted research in the field of Optics [22, 23]. Caliskan and Bilici gave some properties for the involute-evolute curve pair using Frenet frame in Euclidean 3-space [24]. In addition, the pair of involute-evolute curves was investigated in different spaces by using different frames in $[5,6,25-27]$.

The paper is organized as follows. In Sec. 2 we first introduce the notations that we are going to use and give a brief summary of basic definitions and theorems related with curves and surfaces theory in Euclidean 3-space. Moreover, we mention about intrinsic equations of a moving space curve in $\mathbb{E}^{3}$. Then, in the last section, we give some definitions of new type ruled surfaces obtained by using the evolution of involute-evolute curve pair in Euclidean 3-space. Also, we give some nice results for the Gaussian curvature and the mean curvature of these surfaces. Finally, as an application, considering on a given involute-evolute curve pair, we plot the graphics of these new type surfaces using Mathematica.

\section{Preliminaries}

In this section, we would like to give a brief summary of basic definitions, facts and equations in the theory of curves and surfaces in Euclidean 3-space (see for more detail, [1, 2, 7]).

Received : 10-06-2020, Accepted : 27-10-2020 
Let $\mathbb{E}^{3}$ denotes the three-dimensional Euclidean space, i.e., the real vector space $\mathbb{R}^{3}$ endowed with the Riemann metric

$$
\langle,\rangle=d \xi_{1}^{2}+d \xi_{2}^{2}+d \xi_{3}^{2}
$$

where $\left(\xi_{1}, \xi_{2}, \xi_{3}\right)$ is rectangular coordinate system of $\mathbb{E}^{3}$. Thus, the norm of a vector $u \in E^{3}$ is $\|u\|=\sqrt{\langle u, u\rangle}$. Also, for an arbitrary curve $\alpha$ in $\mathbb{E}^{3}$, if $\left\|\alpha^{\prime}(s)\right\|=1$, then the curve $\alpha$ is called as a unit speed curve. Now, let $\alpha$ be a unit speed curve ( or parametrized by arc-length parameter $s$ ) in Euclidean 3-space, $E^{3}$. We denote by $\{T(s), N(s), B(s)\}$ the moving Frenet frame along the curve $\alpha$. Then $T, N$ and $B$ the tangent, the principal normal and the binormal vector of the curve $\alpha$, respectively. Thus the following Frenet formulas hold

$$
\begin{aligned}
T_{s} & =\kappa N, \\
N_{s} & =-\kappa T+\tau B, \\
B_{s} & =-\tau N
\end{aligned}
$$

where $\kappa$ is the curvature, $\tau$ is the torsion.

Definition 2.1. Let $\alpha$ and $\alpha^{*}$ be any two curves in Euclidean 3-space, $\mathbb{E}^{3}$ corresponding with Frenet frames $\{T, N, B\}$ and $\left\{T^{*}, N^{*}, B^{*}\right\}$, respectively. If $\left\langle T, T^{*}\right\rangle=0$, then the curve $\alpha$ is called as involute of the curve $\alpha^{*}$ and also the curve $\alpha^{*}$ is called as evolute of the curve $\alpha$ [1].

This definition suffices to define this curve mate as

$$
\alpha(s)=\alpha^{*}(s)+\lambda(s) T^{*}(s),
$$

where $\lambda(s)=c-s, c \in \mathbb{R}$, [1]. Now, we would like to give some obtained relationship between involute-evolute curve pairs as following:

Theorem 2.1. [3] Let $\alpha$ and $\alpha^{*}$ be the involute-evolute curves with the Frenet frame $\{T, N, B\}$ and $\left\{T^{*}, N^{*}, B^{*}\right\}$, respectively. Then, the relationship between the Frenet frames of the curves $\alpha$ and $\alpha^{*}$ is

$$
\begin{aligned}
T & =N^{*}, \\
N & =\frac{1}{\sqrt{\kappa^{* 2}+\tau^{* 2}}}\left(-\kappa^{*} T^{*}+\tau^{*} B^{*}\right), \\
B & =\frac{1}{\sqrt{\kappa^{* 2}+\tau^{* 2}}}\left(\tau^{*} T^{*}+\kappa^{*} B^{*}\right) .
\end{aligned}
$$

Theorem 2.2. [3] Let $\alpha$ and $\alpha^{*}$ be the involute-evolute curves with the Frenet frames $\{T, N, B\}$ and $\left\{T^{*}, N^{*}, B^{*}\right\}$, respectively. Then, the relationship between the curvature and the torsion of the curves $\alpha$ and $\alpha^{*}$ is

$$
\begin{aligned}
\kappa & =\frac{\sqrt{\kappa^{* 2}+\tau^{* 2}}}{|(-s+c)| \kappa^{*}}, \\
\tau & =\frac{\kappa^{*} \tau_{s}^{*}-\kappa_{s}^{*} \tau^{*}}{(-s+c) \kappa^{*}\left(\kappa^{* 2}+\tau^{* 2}\right)}
\end{aligned}
$$

where $c$ is a real constant and $s$ is the arc-length parameter.

Now, let $\Pi$ be a simply-connected domain in the plane, $\mathbb{E}^{2}$ and $\varphi: \Pi \longrightarrow \mathbb{E}^{3}$ be an immersion in $\mathbb{E}^{3}$. If $\varphi(s, v)$ is a parametrization of surface in $\mathbb{E}^{3}$, then the normal vector field of the surface is given by as

$$
U=\frac{\varphi_{s} \wedge \varphi_{v}}{\left\|\varphi_{s} \wedge \varphi_{v}\right\|}
$$

where $\varphi_{s}=\frac{\partial \varphi}{\partial s}$ and $\varphi_{v}=\frac{\partial \varphi}{\partial v}$. The first fundamental form of the surface $\varphi(s, t)$ is given by

$$
I=\langle d \varphi, d \varphi\rangle=E d s^{2}+2 F d s d v+G d v^{2},
$$

where the coefficients of the first fundamental form are

$$
E=\left\langle\varphi_{s}, \varphi_{s}\right\rangle, \mathrm{F}=\left\langle\varphi_{s}, \varphi_{v}\right\rangle, \mathrm{G}=\left\langle\varphi_{v}, \varphi_{v}\right\rangle
$$


The second fundamental form of the surface $\varphi(s, v)$ is given by

$$
I I=\langle d \varphi, d U\rangle=e d s^{2}+2 f d s d v+g d v^{2},
$$

where

$$
e=\left\langle\varphi_{s s}, U\right\rangle, f=\left\langle\varphi_{s v}, U\right\rangle, \mathrm{g}=\left\langle\varphi_{v v}, U\right\rangle .
$$

Moreover, the Gaussian curvature and the mean curvature of the surface $\varphi(s, v)$ are defined by

$$
K=\frac{e g-f^{2}}{E G-F^{2}} \quad \text { and } \quad H=\frac{1}{2} \frac{E g-2 F f+G e}{E G-F^{2}},
$$

respectively. As in the Euclidean 3-space, the surfaces with vanishing Gaussian curvature are called flat and the surfaces with vanishing mean curvature are called minimal. Moreover, the surfaces with zero Gaussian curvature are named as developable surface.

\subsection{Intrinsic equations of a moving space curve}

In this section, we would like to mention about the evolution of a moving space curve in Euclidean 3-space and so we give the intrinsic equations expressing with curvatures of it corresponding with moving Frenet frame $\{T, N, B\}$, [13]. Let $\alpha$ be a moving space curve described in parametric form by a position vector $\alpha(s, t)$ in Euclidean 3 -space where $s$ is the arc-length parameter and $t$ is the time parameter. Thus, the moving Frenet frame $\{T, N, B\}$ of the curve with respect to $s$ and $t$ can be written in matrix form as follows:

$$
\left[\begin{array}{l}
T \\
N \\
B
\end{array}\right]_{s}=\left[\begin{array}{ccc}
0 & \kappa & 0 \\
-\kappa & 0 & \tau \\
0 & -\tau & 0
\end{array}\right]\left[\begin{array}{l}
T \\
N \\
B
\end{array}\right] \text { and }\left[\begin{array}{c}
T \\
N \\
B
\end{array}\right]_{t}=\left[\begin{array}{ccc}
0 & \nu & \eta \\
-\nu & 0 & \mu \\
-\eta & -\mu & 0
\end{array}\right]\left[\begin{array}{l}
T \\
N \\
B
\end{array}\right]
$$

Here $\nu, \eta$ and $\mu$ are some smooth functions of $s$ and $t$. Now, by using the compatible conditions $T_{s t}=T_{t s}, N_{s t}=N_{t s}$ and $B_{s t}=B_{t s}$, one can obtain

$$
\left(\begin{array}{ccc}
0 & \left(\kappa_{t}-\nu_{s}+\tau \eta\right) & \left(\eta_{s}-\kappa \mu+\tau \nu\right) \\
-\left(\kappa_{t}-\nu_{s}+\tau \eta\right) & 0 & \left(\tau_{t}-\mu_{s}-\kappa \eta\right) \\
-\left(\eta_{s}-\kappa \mu+\tau \nu\right) & -\left(\tau_{t}-\mu_{s}-\kappa \eta\right) & 0
\end{array}\right)=0_{3 \times 3}
$$

From here, the compatibility conditions become

$$
\begin{aligned}
& \kappa_{t}=\nu_{s}-\tau \eta, \\
& \tau_{t}=\mu_{s}+\kappa \eta, \\
& \eta_{s}=\kappa \mu-\tau \nu .
\end{aligned}
$$

As a result, the curvatures of the evolving curve are given as

$$
\begin{aligned}
\kappa_{t} & =\nu_{s}-\tau \eta, \\
\tau_{t} & =\left(\frac{\eta_{s}+\tau \nu}{\kappa}\right)_{s}+\kappa \eta .
\end{aligned}
$$

Thus, according to the Frenet frame, these equations represent the motion of the curve [13].

\section{On Evolution of Some Special Type Ruled Surfaces}

As well knowing, a surface, which can be swept by moving a line in space, is called as a ruled surface [4]. So it has a parameterization given by the following form

$$
\varphi(s, v)=\alpha(s)+v r(s),
$$

where $\alpha(s)$ is called the base curve, $r(s)$ is the director curve and $s, v$ parameters, $u \in I \subset \mathbb{R}, v \in \mathbb{R}$. By moving with time $t$ the curves $\alpha(s)$ and $r(s)$, one can get the evolution equation of the ruled surface given as

$$
\varphi(s, v, t)=\alpha(s, t)+v r(s, t) .
$$

Now we would like to give the following definition: 
Definition 3.1. [16] A surface of evolution $\varphi(s, v, t)$ and its flow $\frac{\partial \varphi}{\partial t}$ are said to be inextensible if its first fundamental quantities $\{E, F, G\}$ satisfies

$$
\frac{\partial E}{\partial t}=\frac{\partial F}{\partial t}=\frac{\partial G}{\partial t}=0
$$

Let $\left\{\alpha, \alpha^{*}\right\}$ be involute-evolute curve pairs in $\mathbb{E}^{3}$ defined as in Definition 2.1. In this section, we would like to investigate some properties of the evolution of three different type ruled surfaces arising from the choose of the director $r(s)$ by the following:

\subsection{Associated Tangent Ruled Surface}

Let us consider on a ruled surface generated by the motion of the tangent vector $T^{*}=r(s)$ of evolute curve $\alpha^{*}$, called as the associated tangent ruled surface $\varphi_{T}$. So from (3.2), the evolution equation of this surface is rewrite as

$$
\varphi_{T}(s, v, t)=\alpha(s, t)+v T^{*}(s, t) .
$$

Then, we have

$$
\begin{aligned}
& \varphi_{T s}(s, v, t)=\left(1+v \kappa^{*}\right) N^{*}(s, t), \\
& \varphi_{T v}(s, v, t)=T^{*}(s, t)
\end{aligned}
$$

where the subscripts $s$ and $v$ represent partial derivatives of the associated tangent ruled surface $\varphi_{T}$. By substituting the above equations into (2.2), the unit normal vector field of this surface is calculated by

$$
U_{T}=\frac{\varphi_{T s} \wedge \varphi_{T v}}{\left\|\varphi_{T s} \wedge \varphi_{T v}\right\|}=-B^{*}(s, t)
$$

On the other hand, by considering (3.5) in (2.3), we obtain the coefficients of the first fundamental form of the associated tangent ruled surface $\varphi_{T}$ as

$$
E_{T}=\left(1+v \kappa^{*}\right)^{2}, F_{T}=0 \text { and } G_{T}=1 .
$$

By considering the above coefficients into the Eq. 3.3, one can see that the associated tangent ruled surface is inextensible if

$$
\kappa^{*}=-\frac{1}{v} \quad \text { or } \quad \kappa_{t}=0 .
$$

Furthermore, by deriving of equations given in (3.5), one can get

$$
\begin{aligned}
& \varphi_{T s s}(s, v, t)=-\kappa^{*}\left(1+v \kappa^{*}\right) T^{*}(s, t)+v \kappa_{s}^{*} N^{*}(s, t)+\tau^{*}\left(1+v \kappa^{*}\right) B^{*}(s, t), \\
& \varphi_{T s v}(s, v, t)=\kappa^{*} N^{*}(s, t), \\
& \varphi_{T v v}(s, v, t)=0 .
\end{aligned}
$$

From the equations (2.4), (3.6) and (3.9), the coefficients of the second fundamental form of the normal surface are given by

$$
e_{T}=-\tau^{*}\left(1+v \kappa^{*}\right), f_{T}=0 \text { and } g_{T}=0 .
$$

Proposition 3.1. The Gaussian curvature and the mean curvature of the associated tangent ruled surface $\varphi_{T}$ are

$$
K_{T}=0 \quad \text { and } \quad H_{T}=\frac{-\tau^{*}}{2\left(1+v \kappa^{*}\right)},
$$

respectively.

Proof. By substituting (3.7) and (3.10) into (2.6), one can readily obtain the Gaussian curvature and the mean curvature of $\varphi_{T}$ as above.

Remark 3.1. 1. The associated tangent ruled surface $\varphi_{T}$ is developable.

2. The associated tangent ruled surface $\varphi_{T}$ is minimal surface if $\tau^{*}=0$. 


\subsection{Associated Normal Ruled Surface}

Secondly, we will take $r(s)=N^{*}$. So, we consider on the ruled surface generated by the motion of the principal normal vector $N^{*}$ of involute curve $\alpha^{*}$, called as the associated normal ruled surface $\varphi_{N}$. As defining of (3.4), one can rewritten the evolution equation of this surface as following

$$
\varphi_{N}(s, v, t)=\alpha(s, t)+v N^{*}(s, t) .
$$

Then, we have

$$
\begin{aligned}
& \varphi_{N s}(s, v, t)=-v \kappa^{*} T^{*}(s, t)+N^{*}(s, t)+v \tau^{*} B^{*}(s, t), \\
& \varphi_{N v}(s, v, t)=N^{*}(s, t),
\end{aligned}
$$

respectively. In addition, by using (2.2), we obtain

$$
U_{N}=-\frac{\tau^{*}}{\sqrt{\kappa^{* 2}+\tau^{* 2}}} T^{*}(s, t)-\frac{\kappa^{*}}{\sqrt{\kappa^{* 2}+\tau^{* 2}}} B^{*}(s, t) .
$$

Now by combining (3.13) with (2.3), we get

$$
E_{N}=1+v^{2}\left(\kappa^{* 2}+\tau^{* 2}\right), F_{N}=1 \text { and } G_{N}=1 .
$$

Now, considering these coefficients into the Eq. 3.3, one can see that the associated normal ruled surface is inextensible if

$$
\kappa^{*} \kappa_{t}^{*}+\tau^{*} \tau_{t}^{*}=0 .
$$

By deriving of the equations in (3.13), we get

$$
\begin{aligned}
& \varphi_{N s s}(s, v, t)=\left(-\kappa^{*}-v \kappa_{s}^{*}\right) T^{*}(s, t)-v\left(\kappa^{* 2}+\tau^{* 2}\right) N^{*}(s, t)+\left(\tau^{*}+v \tau_{s}^{*}\right) B^{*}(s, t), \\
& \varphi_{N s v}(s, v, t)=-\kappa^{*} T^{*}(s, t)+\tau^{*} B^{*}(s, t), \\
& \varphi_{N v v}(s, v, t)=0 .
\end{aligned}
$$

By combining (3.14) and (3.17) with (2.4), we obtain

$$
e_{N}=\frac{v\left(\kappa_{s}^{*} \tau^{*}-\kappa^{*} \tau_{s}^{*}\right)}{\sqrt{\kappa^{* 2}+\tau^{* 2}}}, f_{N}=0 \text { and } g_{N}=0 .
$$

Proposition 3.2. The Gaussian curvature and the mean curvature of the associated normal ruled surface $\varphi_{N}$ are given by

$$
K_{N}=0 \quad \text { and } \quad H_{N}=\frac{\left(\kappa_{s}^{*} \tau^{*}-\kappa^{*} \tau_{s}^{*}\right)}{2\left(\kappa^{* 2}+\tau^{* 2}\right)^{\frac{3}{2}}}
$$

respectively.

Proof. By substituting (3.15) and (3.18) into the equations (2.6), we directly obtain the Gaussian curvature and the mean curvature of a normal ruled surface $\varphi_{N}$ as above.

From these equations, the following result is obvious.

Remark 3.2. 1. The associated normal ruled surface $\varphi_{N}$ is developable.

2. The associated normal ruled surface $\varphi_{N}$ is minimal surface if $\frac{\kappa^{*}}{\tau^{*}}$ is constant and $\tau^{*} \neq 0$.

\subsection{Associated Binormal Ruled Surface}

Finally, we will take $r(s)=B^{*}$. Thus, we will consider on the ruled surface generated by the motion of the binormal vector $B^{*}$ of involute curve $\alpha^{*}$, called as the associated binormal ruled surface $\varphi_{B}$. As defining of (3.4), one can rewritten the evolution equation of this surface as following

$$
\varphi_{B}(s, v, t)=\alpha(s, t)+v B^{*}(s, t) .
$$


Then, we have

$$
\begin{aligned}
& \varphi_{B s}(s, v, t)=\left(1-v \tau^{*}\right) N^{*}(s, t), \\
& \varphi_{B v}(s, v, t)=B^{*}(s, t) .
\end{aligned}
$$

Morever, by using (2.2), we get the unit normal field of this surface as

$$
U_{B}=\frac{\varphi_{B s} \wedge \varphi_{B v}}{\left\|\varphi_{B s} \wedge \varphi_{B v}\right\|}=T^{*}(s, t) .
$$

By combining (3.20) with (2.3), then we obtain

$$
E_{B}=\left(1-v \tau^{*}\right)^{2}, F_{B}=0 \text { and } G_{B}=1 .
$$

Now, by considering these coefficients into the Eq. 3.3, one can see that the associated binormal ruled surface is inextensible if

$$
\tau^{*}=-\frac{1}{v} \quad \text { or } \quad \tau_{t}=0 .
$$

From the equation (3.20), the second derivative is found and given as

$$
\begin{aligned}
& \varphi_{B s s}(s, v, t)=-\kappa^{*}\left(1-v \tau^{*}\right) T^{*}(s, t)-v \tau^{*} N^{*}(s, t)+\tau^{*}\left(1-v \tau^{*}\right) B^{*}(s, t), \\
& \varphi_{B s v}(s, v, t)=-\tau^{*} N^{*}(s, t), \\
& \varphi_{B v v}(s, v, t)=0 .
\end{aligned}
$$

By substituting (3.21) and (3.24) into (2.4), then we get

$$
e_{B}=-\kappa^{*}\left(1-v \tau^{*}\right), f_{B}=0 \text { and } g_{B}=0 .
$$

Proposition 3.3. For the binormal ruled surface $\varphi_{B}$, the Gaussian and mean the curvatures are

$$
K_{B}=0 \quad \text { and } \quad H_{B}=-\frac{\kappa^{*}}{2\left(1-v \tau^{*}\right)},
$$

respectively.

Proof. By substituting (3.22) and (3.25) into (2.6), one can readily obtain the Gaussian curvature and the mean curvature of $\varphi_{B}$ as above.

From these equations, the following result is obvious.

Remark 3.3. 1. The associated binormal ruled surface $\varphi_{B}$ is developable.

2. The associated binormal ruled surface $\varphi_{B}$ is minimal surface if $\kappa^{*}=0$.

\section{Applications}

Example 4.1. Let $\alpha$ and $\alpha^{*}$ be the involute-evolute curves as defined in (2.1). By considering the unit speed curve $\alpha(s):(-\pi, \pi) \rightarrow E^{3}$ given by

$$
\alpha(s)=\frac{3}{4}\left(\cos (s)+\frac{\cos (3 s)}{9}, \sin (s)+\frac{\sin (3 s)}{9}, \frac{-2 \cos (s)}{\sqrt{3}}\right),
$$

we have the Frenet apparatus of the curve $\alpha$ is given by

$$
\left\{\begin{array}{c}
T=\frac{3}{4}\left(-\sin (s)-\frac{\sin (3 s)}{3}, \cos (s)+\frac{\cos (3 s)}{3}, \frac{2 \sin (s)}{\sqrt{3}}\right), \\
N=\left(-\frac{\sqrt{3} \cos (2 s)}{2},-\frac{\sqrt{3} \sin (2 s)}{2}, \frac{1}{2}\right), B=\left(\frac{3 \cos (s)-\cos (3 s)}{4}, \sin (s)^{3}, \frac{\sqrt{3} \cos (s)}{2}\right), \\
\kappa=\sqrt{3} \cos (s), \tau=\sqrt{3} \sin (s)
\end{array}\right\} .
$$

By taking $c=1$ in (2.1) and considering (4.1), we get the involute curve $\alpha^{*}$ of $\alpha$ such that

$$
\alpha^{*}(s)=\left(\begin{array}{c}
\frac{1}{12}(9 \cos (s)+\cos (3 s)+3(-1+s)(3 \sin (s)+\sin (3 s))) \\
\frac{1}{12}(3(1-s)(3 \cos (s)+\cos 3 s)+9 \sin (s)+\sin (3 s)), \\
-\frac{\sqrt{3}}{2}(\cos (s)+(-1+s) \sin (s)) .
\end{array}\right) .
$$




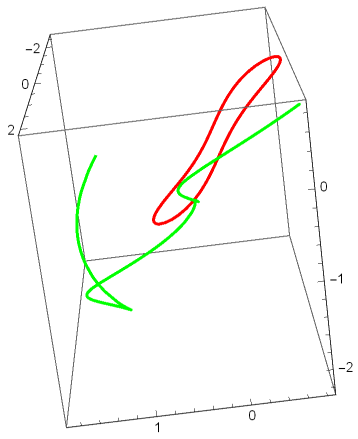

Figure 1. The curve $\alpha$ as in (4.1) given with in green and the involute curve $\alpha^{*}$ as in (4.2) given with in red.

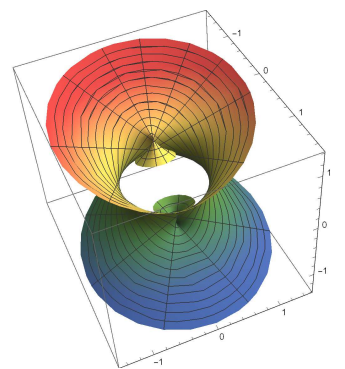

Figure 2. The graph of associated tangent ruled surface $\varphi_{T}$ as in (4.4) with $s \in(2,10)$ and $v \in(-1,1)$.

(See Fig. 1). So, the Frenet apparatus $\left\{T^{*}, N^{*}, B^{*}, \kappa^{*}, \tau^{*}\right\}$ of the curve $\alpha^{*}$ is given by

$$
\left\{\begin{array}{c}
T^{*}=\left(-\frac{\sqrt{3} \cos (2 s)}{2},-\frac{\sqrt{3} \sin (2 s)}{2}, \frac{1}{2}\right), \\
N^{*}=(\sin (2 s), \cos (2 s), 0), B^{*}=\left(\frac{\cos (2 s)}{2}, \frac{\sin (2 s)}{2}, \frac{\sqrt{3}}{2}\right) \\
\kappa^{*}=\frac{\sec s}{(s-1)}, \tau^{*}=\frac{\sec s}{\sqrt{3}(s-1)}
\end{array}\right\} .
$$

Now by considering the curve $\alpha$ and the Frenet vectors $\left\{T^{*}, N^{*}, B^{*}\right\}$ of the involute curve $\alpha^{*}$ as defined above into (3.4), (3.12) and (3.19), respectively, then the parametric equations of the associated tangent ruled surface, associated normal ruled surface and associated binormal ruled surface are obtained by

$$
\begin{gathered}
\varphi_{T}(s, v)=\left(\frac{3}{4}\left(\cos (s)+\frac{\cos (3 s)}{9}\right)-\frac{v \sqrt{3} \cos (2 s)}{2}, \frac{3}{4}\left(\sin (s)+\frac{\sin (3 s)}{9}\right)-\frac{v \sqrt{3} \sin (2 s)}{2}, \frac{-\sqrt{3} \cos (s)}{2}+\frac{v}{2}\right), \\
\varphi_{N}(s, v)=\left(\frac{3}{4}\left(\cos (s)+\frac{\cos (3 s)}{9}\right)+v \sin (2 s), \frac{3}{4}\left(\sin (s)+\frac{\sin (3 s)}{9}\right)+v \cos (2 s), \frac{-\sqrt{3} \cos (s)}{2}\right),
\end{gathered}
$$

and

$$
\varphi_{B}(s, v)=\left(\frac{3}{4}\left(\cos (s)+\frac{\cos (3 s)}{9}\right)+\frac{v \cos (2 s)}{2}, \frac{3}{4}\left(\sin (s)+\frac{\sin (3 s)}{9}\right)+\frac{v \sin (2 s)}{2}, \frac{-\sqrt{3} \cos (s)}{2}+\frac{v \sqrt{3}}{2}\right),
$$

respectively. Now, we would like to give some explicit examples of the associated ruled surfaces in $\mathbb{E}^{3}$ :

\section{Acknowledgements}

The authors would like to thank the referees for their valuable and useful comments that further improve the article. 


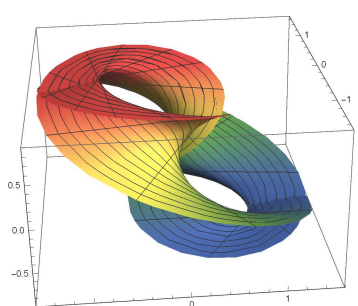

Figure 3. The graph of associated normal ruled surface $\varphi_{N}$ as in (4.5) with $s \in(2,10)$ and $v \in(-1,1)$.

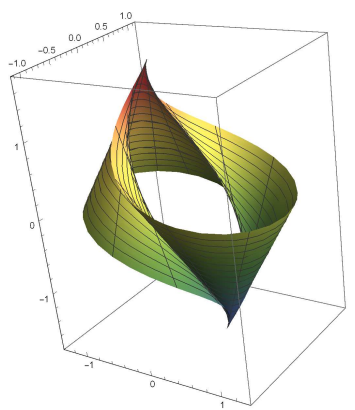

Figure 4. The graph of associated binormal ruled surface $\varphi_{B}$ as in (4.6) with $s \in(2,10)$ and $v \in(-1,1)$.

\section{References}

[1] Hacisalihoglu, H. H.: Differential geometry. Ankara University Faculty of Science Press. Ankara (2000).

[2] Kuhnel, W.: Differential geometry: curves-surfaces-manifolds. Braunschweig. Wiesbaden (1999).

[3] Sabuncuoğlu, A.: Differential geometry. Nobel Press. Ankara (2014).

[4] Ergut, M.: The drall and the scalar normal curvatures of $(r+1)$-dimensional generalized ruled surfaces. Commun. Fac. Sci. Univ. Ank. 38, 115-125 (1989).

[5] Yilmaz, M.Y., Bektas, M., Ergut, M.: The pair of involute-evolute curves in Finsler manifold F3. Pure and Appl. Maths J. of Inst. of Kyrgyzstan National Academy, No. 39, Bishkek, (2008), 156-163.

[6] Isah, MA., Kulahci, M.A.: Involute Curves in 4-Dimensional Galilean Space G4. Conference Proceedings of Science and Technology, 2(2), (2019), 134a141.

[7] Do Carmo, M.: Differential geometry of curves and surfaces. Prentice-Hall. Englewood Cliffs (1976).

[8] Gray, A.: Modern differential geometry of curves and surfaces with mathematica. CRC. New York (1998).

[9] Nakayama, K., Wadati, M.: Motion of curves in the plane. J Phys. Soc. Japan. 62, 473-479 (1993).

[10] Abdel-All, N. H., Hussien, R. A., Youssef, T.: Evolution of curves via the velocities of the moving frame. J. Math. Comput. Sci. 2, 1170-1185 (2012).

[11] Doliwa, A., Santini, P.: An elementary geometric characterization of the integrable motions of a curve. Physics Letters A. 185, 373-384 (1994).

[12] Kwon, D. Y., Park, F. C.: Evolution of inelastic plane curves. Applied Mathematics Letters. 12, 115-119 (1999).

[13] Rogers, C., Schief, W. K.: Bäcklund and Darboux transformations: Geometry and modern applications in soliton theory. Cambridge University Press (2002).

[14] Abd-Ellah, H. N.: Evolution of translation surfaces in Euclidean 3-space. Applied Mathematics \& Information Sciences. 9, 661-668 (2015).

[15] Hussien, R. A., Mohamed, S. G.: Generated surfaces via inextensible flows of curves in $\mathrm{R}^{3}$. Journal of Applied Mathematics. Article ID: 6178961 (2016). 
[16] Kwon, D. Y., Park, F. C.: Inextensible flows of curves and developable surfaces. Applied Mathematics Letters. 18, 1156-1162 (2005).

[17] Yildiz, O. G., Ersoy, S., Masal, M.: Note on inextensible flows of curves on oriented surface. CUBO A Mathematical Journal. 16 (3), 11-19 (2014).

[18] Nakayama, K., Wadati, M.: The motion of surfaces. J. Phys. Soc. of Japan. 62, 1895-1901 (1993).

[19] Hussien, R. A., Youssef, T.: Evolution of special ruled surfaces via the evolution of their directrices in Euclidean 3-space $\mathbb{E}^{3}$. Applied Mathematics \& Information Sciences. 10, 1949-1956 (2016).

[20] Soliman, M. A., Abdel-All, N. H., Hussien, R. A., Youssef, T.: Evolutions of the ruled surfaces via the evolution of their directrix using quasi frame along a space curve. Journal of Applied Mathematics and Physics. 6 (8), 1748-1756 (2018).

[21] Eren, K., Koksal, H. H.: Evolution of space curves and the special ruled Surfaces with modified orthogonal frame. AIMS Mathematics. 5 (3), 2027a2039 (2020).

[22] As, E., Sarioglugil, A.: On the Bishop curvatures of involute-evolute curve couple in $\mathbb{E}^{3}$. International Journal of Physical Sciences. 9(7), 140a145 (2014).

[23] Boyer, C.: A History of mathematics. New York:Wiley. p. 334 (1968).

[24] Caliskan, M., Bilici, M.: Some characterizations for the pair of involute evolute curves in Euclidean space $\mathbb{E}^{3}$. Bulletin of Pure and Applied Sciences. 2 E(2), 289-294 (2002).

[25] Akyigit, M., Azak, A.Z., Ersoy, S.: Involute-evolute curves in Galilean space $\mathbb{G}^{3}$. Scientia Magna. 6 (4), 75-80 (2010).

[26] Bukcu, B., Karacan, M. K.: On the involute and evolute curves of the spacelike curve with a spacelike binormal in Minkowski 3-space. International Journal of Contemporary Mathematical Sciences. 5 (2), 221-232 (2007).

[27] Senyurt, S., Altun, Y., Cevahir, C.: On the Darboux vector belonging to involute curve a different view. Mathematical Sciences and Applications E-Notes. 4 (2), 131-138 (2016).

[28] Yoon, DW., Yuzbasi, ZK., Aslan, EC.: Evolution of Spacelike Curves and Special Timelike Ruled Surfaces in the Minkowski Space. Preprint arXiv:1908.00053 (2019).

[29] Yuzbasi, ZK., Aslan, EC., Inc, M., Baleanu, D.: On exact solutions for new coupled nonlinear models getting evolution of curves in Galilean space. Thermal Science 23 (Suppl. 1), 227-233 (2019).

\section{Affiliations}

\section{AleV KeLleCi}

ADDRESS: Firat University, Dept. of Mathematics, 23100, Elazig-Turkey.

E-MAIL: alevkelleci@hotmail.com

ORCID ID:0000-0003-2528-2131

\section{KEMAL EREN}

ADDRESS: Sakarya University, Dept. of Math., 54187, Sakarya-Turkey.

E-MAIL: kemaleren52@gmail.com

ORCID ID:0000-0001-5273-7897 